\title{
Concentración iónica y metales pesados en el agua de riego de la cuenca del río Ayuquila-Tuxcacuesco-Armería
}

\author{
Ionic strength and heavy metals in irrigation water basin \\ Ayuquila-Tuxcacuesco-Armeria river

\begin{abstract}
Oscar Raúl Mancilla-Villa1*, Blanca Elizabeth Fregoso-Zamorano², Eva Judith Hueso-Guerrero², Rubén Dario Guevara-Gutiérrez ${ }^{3}$, Carlos Palomera-García3; José Luis Olguín-López;3 Héctor
\end{abstract} \\ Manuel Ortega-Escobar ${ }^{4}$, Edna Karen Medina Valdovinos ${ }^{2}$, Héctor Flores-Magdaleno ${ }^{4}$
}

\begin{abstract}
RESUMEN
Los altos niveles de concentración de metales pesados en agua utilizada para riego representan un problema importante para la agricultura y la salud humana, así como para la biodiversidad, por tal motivo se realizó el presente trabajo en relación con la calidad del agua en la cuenca del río Ayuquila-Tuxcacuesco-Armería. El estudio se realizó entre febrero y abril de 2014, se tomaron 40 muestras de agua. Se analizaron el potencial de hidrógeno, la conductividad eléctrica (CE), aniones y cationes, así como As y metales pesados totales: $\mathrm{Cd}, \mathrm{Hg}$ y $\mathrm{Pb}$. En la distribución de la concentración iónica predominó el anión bicarbonato y el catión magnesio. Los metales pesados se determinaron utilizando la metodología recomendada por la EPA (Environmental Protection Agency) y APHA (American Public Health Association). Se comparó la calidad del agua con los criterios de la NOM-001-ECOL-1996, de EPA (1986) y SEDUE (1989). Los resultados mostraron valores bajos en la concentración de metales pesados en agua para riego agrícola y uso urbano, no así para aguas naturales, pues $100 \%$ de las muestras presentaron concentraciones por encima de los límites permisibles para $\mathrm{Hg}$ y 92,5\% para Pb. La concentración más alta la presentó el $\mathrm{Hg}$ con valores de $0,0039 \mathrm{mg} \mathrm{L}^{-1}$ a $0,0203 \mathrm{mg} \mathrm{L}^{-1}$, mientras que la menor con valores de $0,0007 \mathrm{mg} \mathrm{L}^{-1}$ a $0,0086 \mathrm{mg} \mathrm{L}^{-1}$ fue para el $\mathrm{Pb}$. En cuanto a salinidad, las muestras entraron en las clasificaciones $\mathrm{C} 2, \mathrm{C} 3 \mathrm{y}$ $\mathrm{C} 4$, y en sodicidad todas se clasificaron como S1. En solidos totales disueltos la mayoría presentan valores entre $450-2.000 \mathrm{mg} \mathrm{L}^{-1}$.

Palabras clave: metales pesados, agua de riego, límites permisibles.
\end{abstract}

\section{ABSTRACT}

High levels of heavy metals concentrations in water used for irrigation represent an important agriculture and human health problem as well as for biodiversity, for that reason this paper regarding water quality in the basin was conducted river Ayuquila-TuxcacuescoArmeria. The study was conducted between February and April 2014, 40 water samples were taken. Hydrogen potential analyzed, electrical conductivity (EC), anions, cations and total As and heavy metals $\mathrm{Cd}, \mathrm{Hg}$ and $\mathrm{Pb}$. The bicarbonate anion and the magnesium cation predominated in the distribution of the ionic concentration. Heavy metals were determined using the recommended EPA (Environmental Protection Agency) and APHA (American Public Health Association) methodology. Water quality criteria NOM-001ECOL-1996, EPA (1986) and SEDESOL (1989) was compared. The results showed low values in the concentration of heavy metals in water for agricultural irrigation and urban use, not for natural waters, for 100\% of the samples had levels above the permissible

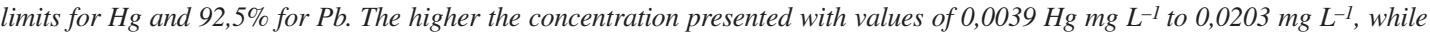
lower values of 0,0007 $\mathrm{mg} \mathrm{L}^{-1}$ to 0,0086 $\mathrm{mg} \mathrm{L}^{-1}$ was for Pb. As for salinity, the samples entered the classifications $C 2, C 3$ and C4, and in sodicity all were classified as S1. In dissolved solids the majority have values between 450-2,000 $\mathrm{mg} \mathrm{L}^{-1}$.

Key words: heavy metals, irrigation water, allowable limits.

1 Departamento de Producción Agrícola del Centro Universitario de la Costa Sur, Universidad de Guadalajara; Av. Independencia Nacional \# 151 C.P. 48900, Autlán de Navarro, Jalisco, México.

2 Centro Universitario de la Costa Sur, Universidad de Guadalajara; Av. Independencia Nacional \# 151 C.P. 48900, Autlán de Navarro, Jalisco, México.

3 Universidad de Guadalajara, Centro Universitario de la Costa Sur, Departamento de Ecología y Recursos Naturales, Av. Independencia Nacional \# 151, Autlán de Navarro, Jal. 48900.

4 Colegio de Postgraduados, Campus Montecillo, carretera México Texcoco, km 36.5. C.P. 56230, Texcoco, México.

* Autor para correspondencia: oscar.mancilla@academicos.udg.mx vios@colpos.mx

Fecha de Recepción: 22 Noviembre, 2016.

Fecha de Aceptación: 16 Junio, 2017. 


\section{Introducción}

En la agricultura de regadío la calidad del agua es un importante factor a considerar para la obtención de altos rendimientos agrícolas. En aguas para uso agrícola la calidad es definida por la concentración de iones específicos, $\mathrm{Ca}^{2+}, \mathrm{Mg}^{2+}$, $\mathrm{Na}^{+}$y K ${ }^{+}$como cationes y $\mathrm{CO}_{3}{ }^{2-}, \mathrm{HCO}_{3}{ }^{-}, \mathrm{Cl}^{-}$y $\mathrm{SO}_{4}{ }^{2-}$ como aniones. Estos elementos tienen su origen en la disolución o meteorización de las rocas y suelos, y son transportados mediante corrientes, tanto superficiales como subterráneas, y depositados en el suelo de forma natural o mediante el riego (Velázquez et al., 2002).

Los metales pesados se encuentran generalmente como componentes naturales de la corteza terrestre, en forma de minerales, sales u otros compuestos. No pueden ser degradados o destruidos fácilmente de forma natural o biológica ya que no tienen funciones metabólicas específicas para los seres vivos (Méndez et al., 2009), agrupan sustancias como cadmio y mercurio, principales contaminantes, además de níquel y plomo, entre otras. Pueden incorporarse a un sistema de abastecimiento de agua por medio de residuos industriales que son vertidos sin previos tratamientos, y posteriormente se depositan en lagos, ríos y distintos sistemas acuíferos (Duffus, 2002). En los cultivos, la acumulación de metales pesados se da por medio de su absorción por el agua de regadío contaminada, por la tierra mediante las raíces o por la deposición en el follaje de partículas aerotransportadas (Mor \& Ceylan, 2008).

Varios autores han mostrado el riesgo de contaminación por metales pesados en el agua (Mancera-Rodríguez et al., 2006; Prieto et al., 2007; Méndez et al., 2012); en la acumulación de metales pesados en el suelo (Martí et al., 2011; Sarabia et al., 2011) y en el riesgo potencial para la salud humana debido a la acumulación de metales pesados en las plantas (Llugany et al., 2007).

El cauce del río Ayuquila y los cuerpos de agua presentes en la cuenca reciben múltiples descargas de tipo industrial, municipal y rural (p.e., granjas porcícolas, granjas bovinas, empresas agrícolas, actividad minera), consideradas fuentes de una gran variedad de contaminantes como son los metales pesados. Se reportan metales pesados en algunos sitios de la cuenca del río Ayuquila; sin embargo, de acuerdo con Mancilla-Villa et al. (2012) las concentraciones encontradas no representan un riesgo para la biodiversidad.
Por otro lado, las concentraciones de cianuro encontradas sí constituían una restricción al aprovechamiento seguro y productivo del agua en uso agrícola y en actividades de recreación con contacto primario, particularmente en la población de El Corcovado. En esta localidad se superó el nivel máximo de $0,02 \mathrm{mg} \mathrm{L}^{-1}$, que aplica para uso recreativo con contacto primario y riego agrícola (SEMARNAT-NOM-001-ECOL-1996). En otro estudio realizado por CONAGUA (2007), se encontró la presencia de la mayoría de los metales pesados a excepción del zinc, arsénico y mercurio, siendo la presencia de cadmio y níquel los más abundantes y de valores más altos siguiéndoles el plomo, cromo y cobre en respectivo orden descendente; en el mismo estudio se encontraron altos valores de cianuros.

El presente estudio aporta información sobre la calidad del agua de riego en la cuenca del río Ayuquila-Tuxcacuesco-Armería, en lo referente a contenido de metales pesados totales, aniones, cationes, conductividad eléctrica y $\mathrm{pH}$.

\section{Materiales y Métodos}

El área de estudio se localiza entre los $102^{\circ} 56^{\prime}$ y $104^{\circ} 35^{\prime}$ longitud oeste $18^{\circ} 40^{\prime}$ y $20^{\circ} 29^{\prime}$ latitud norte, en el occidente de México y forma parte de los estados de Jalisco y Colima (Figura 1). El agua muestreada es utilizada para riego agrícola. Las muestras se tomaron en el río principal y sus afluentes, así como en canales de agua de riego, norias y pozos profundos. Estas se obtuvieron en los municipios de: Tecolotlán, Unión de Tula, Autlán, El Grullo, El Limón, Tonaya, Tolimán, San Gabriel, Tuxcacuesco en Jalisco; Comala, Villa de Álvarez, Armería y Coquimatlán.

La investigación de campo se realizó entre los meses de febrero y abril de 2014. Se localizaron zonas ubicadas dentro de la cuenca donde se utiliza el agua para riego, estableciendo un total de 40 sitios. En cada uno se colectaron dos muestras en recipientes de plástico de $0,5 \mathrm{~L}$, a los que se les colocó una etiqueta con su respectiva fecha, nombre y localización del sitio, para posteriormente realizar las determinaciones en el laboratorio (Tabla 1).

Los metales pesados totales se determinaron con la técnica de espectrometría de emisión atómica, mediante plasma de acoplamiento inductivo con el ICP, modelo Perkin Elmer 5300 Optima, en el 

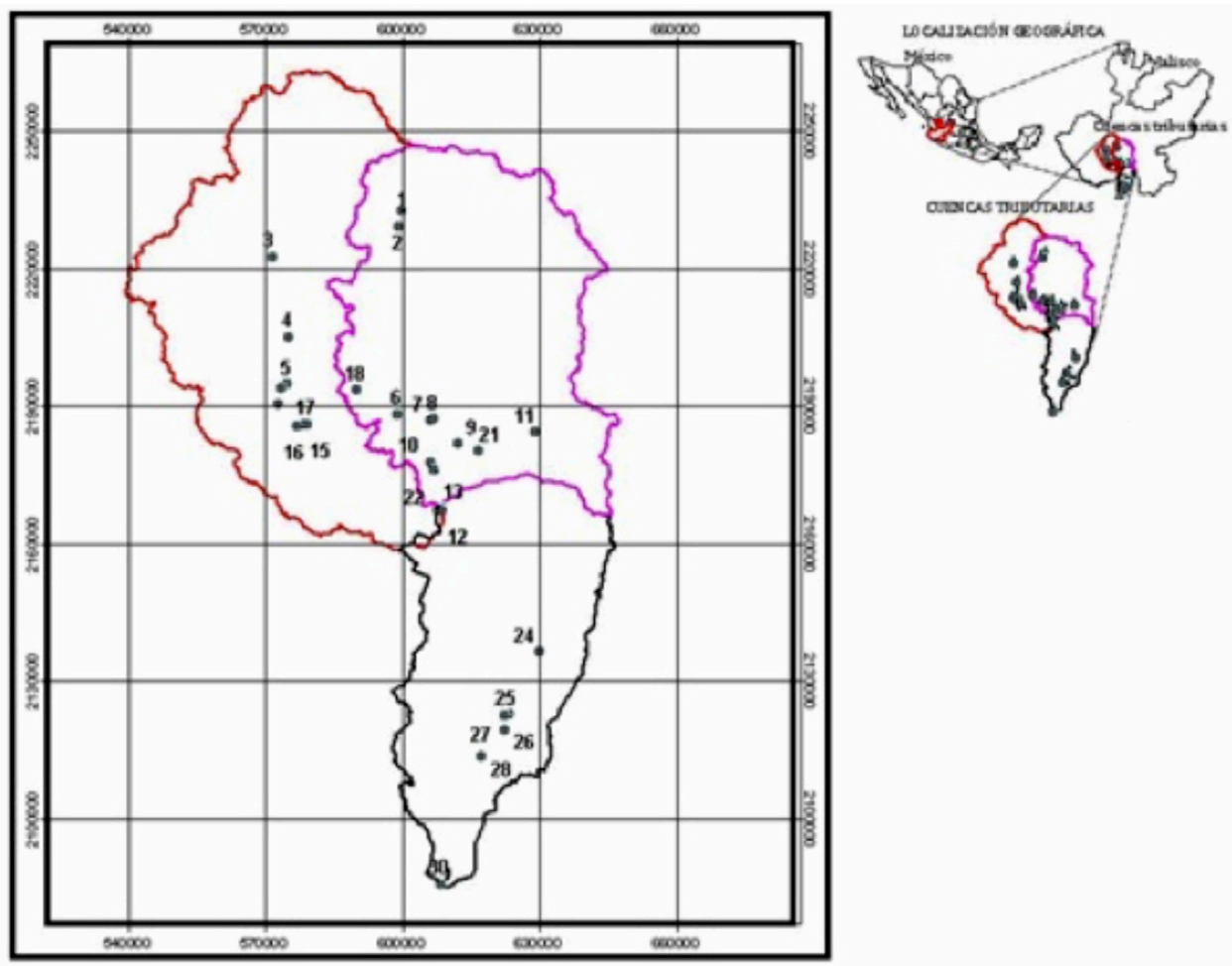

Figura 1. Localización del área de estudio.

Tabla 1. Metodologías necesarias y equipo para las determinaciones fisicoquímicas analíticas de las aguas de riego de la cuenca del río Ayuquila-Tuxcacuesco-Armería.

\begin{tabular}{|c|c|c|c|}
\hline & Determinación & Método & Referencia \\
\hline 1 & $\mathrm{Ph}$ & $\begin{array}{l}\text { Potenciométrico, con Potenciómetro marca Beckman, modelo } \\
\text { Hoffman Pinther Boswork }\end{array}$ & Eaton et al., 1995 \\
\hline 2 & Conductividad eléctrica & $\begin{array}{l}\text { Conductimetría, mediante Conductímetro con puente de } \\
\text { Wheastone con celda de vidrio }\end{array}$ & Richards, 1990 \\
\hline 3 & Sodio y potasio & $\begin{array}{l}\text { Flamometría, mediante Flamómetro IL Autocal Flame } \\
\text { Photometer } 643, \mathrm{~L}=589 \mathrm{~nm} \text {, calibrado con soluciones } \\
\text { estándar de } 145 \mathrm{mmol}_{\mathrm{c}} \mathrm{L}^{-1} \text { para Na y de } 5 \mathrm{mmol}_{\mathrm{c}} \mathrm{L}^{-1} \text { para } \mathrm{K}\end{array}$ & APHA, 1995 3500- $\mathrm{Na}^{+}$y K ${ }^{+}$, D \\
\hline 4 & Calcio y magnesio & $\begin{array}{l}\text { Volumétrico, mediante titulación con EDTA y Eriocromo } \\
\text { Negro T como indicador, para } \mathrm{Ca}+\mathrm{Mg} \text { y Murexida para } \mathrm{Ca}\end{array}$ & APHA, $19953500 \mathrm{Ca}^{2+}, \mathrm{D}$ \\
\hline 5 & Carbonatos & $\begin{array}{l}\text { Volumétrico, mediante titulación con ácido sulfúrico y } \\
\text { Fenolftaleína como indicador }\end{array}$ & APHA, 19952320 B \\
\hline 6 & Bicarbonatos & $\begin{array}{l}\text { Volumétrico, mediante titulación con ácido sulfúrico y } \\
\text { Anaranjado de Metilo como indicador }\end{array}$ & APHA, 19952320 B \\
\hline 7 & Cloruros & $\begin{array}{l}\text { Titulación con Nitrato de Plata y Cromato de Potasio al 5\% } \\
\text { como indicador }\end{array}$ & APHA, 1995 4500-C-IB \\
\hline 8 & Sulfatos & $\begin{array}{l}\text { Turbidimetría, mediante el Espectrofotómetro modelo Perkin } \\
\text { Elmer } 35, \mathrm{~L}=420 \mathrm{~nm}\end{array}$ & APHA, 1995 4500- $\mathrm{SO}_{4}{ }^{2-} \mathrm{E}$ \\
\hline 9 & Metales pesados totales & $\begin{array}{l}\text { Espectrometría de emisión atómica, mediante Plasma de } \\
\text { acoplamiento inductivo con el ICP Perkin Elmer } 5300 \text { Optima, }\end{array}$ & EPA-1983. \\
\hline
\end{tabular}


Laboratorio de Ciencias Ambientales del Colegio de Posgraduados, Campus Montecillo; los metales pesados analizados fueron $\mathrm{As}, \mathrm{Cd}, \mathrm{Hg}$ y $\mathrm{Pb}$. A las muestras se les agregó ácido nítrico concentrado a $\mathrm{pH}<2$, con base en los lineamientos de EPA (1983) y APHA (1995).

Los valores de recuperación obtenidos en las muestras fortificadas, en agua desionizada, para el análisis de los metales fueron los siguientes: As $108,17 \%, \mathrm{Hg} 91,91 \%$, Cd 108,93\% y $\mathrm{Pb} 101,02 \%$; dichos valores se encuentran dentro del rango recomendado y cercanos al 100\% (EPA, 1996).

Para evaluar la calidad del agua del río Ayuquila-Tuxcacuesco-Armería, se utilizaron los criterios de EPA (1986), la SEMARNAT-NOM-001ECOL-1996 y los criterios ecológicos de calidad de agua para diferentes usos (SEDUE, 1989), que establecen los límites máximos permisibles para metales pesados contenidos en el agua para uso agrícola y urbano (Tabla 2).

\section{Resultados y Discusión}

En la Tabla 3, se presenta la ubicación geográfica, la altitud y los nombres de los sitios de muestreo que fueron considerados en la presente investigación.

En la Figura 2, se presenta la distribución de la concentración iónica de las aguas de riego

Tabla 2. Límites máximos permisibles para metales pesados.

\begin{tabular}{|c|c|c|c|c|c|c|c|c|}
\hline \multirow{2}{*}{$\begin{array}{l}\text { Parámetro } \\
\left(\mathrm{mg} \mathrm{L}^{-1}\right)\end{array}$} & \multicolumn{2}{|c|}{$\begin{array}{c}\text { Ríos uso en riego } \\
\text { agrícola }\end{array}$} & \multicolumn{2}{|c|}{$\begin{array}{l}\text { Ríos uso público } \\
\text { urbano }^{\mp}\end{array}$} & \multirow{2}{*}{$\begin{array}{c}\text { Aguas } \\
\text { naturales } \\
(\mathrm{EPA}, 1986)\end{array}$} & \multirow{2}{*}{$\begin{array}{c}\text { Riego } \\
\text { agrícola } \\
\text { (SEDUE, } \\
\text { 1989) }\end{array}$} & \multirow{2}{*}{$\begin{array}{c}\text { Fuente de } \\
\text { abastecimiento } \\
\text { de agua potable } \\
\text { (SEDUE, 1989) }\end{array}$} & \multirow{2}{*}{$\begin{array}{l}\text { Recreativo } \\
\text { e Industrial } \\
\text { (SEDUE, } \\
1989 \text { ) }\end{array}$} \\
\hline & $\begin{array}{c}\text { Promedio } \\
\text { mensual }\end{array}$ & $\begin{array}{l}\text { Promedio } \\
\text { diario }\end{array}$ & $\begin{array}{c}\text { Promedio } \\
\text { mensual }\end{array}$ & $\begin{array}{l}\text { Promedio } \\
\text { diario }\end{array}$ & & & & \\
\hline Arsénico & 0,2 & 0,4 & 0,1 & 0,2 & 0,05 & 0,1 & 0,05 & - \\
\hline Cadmio & 0,2 & 0,4 & 0,1 & 0,2 & 0,01 & 0,01 & 0,01 & 0,01 \\
\hline Mercurio & 0,01 & 0,02 & 0,01 & 0,01 & 0,002 & - & - & - \\
\hline Plomo & 0,5 & 1 & 0,2 & 0,4 & 0,0015 & 5 & 0,05 & 0,1 \\
\hline
\end{tabular}

Fuente: NOM-001-ECOL-1996; Mancilla-Villa, 2012a.

¥ Valores idénticos para protección de vida acuática.

Tabla 3. Localización de las zonas de muestreo de las aguas de riego del río Ayuquila-Tuxcacuesco-Armería.

\begin{tabular}{|c|c|c|c|c|c|c|c|c|}
\hline \multirow{2}{*}{$\mathrm{N}^{\mathrm{o}}$} & \multirow{2}{*}{ Lugar } & \multirow{2}{*}{$\frac{\text { Posición geográfica }}{\text { Lat. Norte Long. Oeste }}$} & \multirow{2}{*}{$\begin{array}{l}\text { Altitud } \\
\text { (m) }\end{array}$} & \multirow{2}{*}{$\mathrm{N}^{\mathrm{o}}$} & \multirow{2}{*}{ Lugar } & \multicolumn{2}{|c|}{ Posición geográfica } & \multirow{2}{*}{$\begin{array}{l}\text { Altitud } \\
(\mathrm{m})\end{array}$} \\
\hline & & & & & & Lat. Norte & Long. Oeste & \\
\hline 1 & Río Tecolotlán & $20^{\circ} 1123.30104^{\circ} 0223.40$ & & 21 & Noria 1 Tuxcacuesco & $19^{\circ} 4046.30$ & $103^{\circ} 5838.90$ & 708 \\
\hline 2 & Presa El Pochote & $20^{\circ} 1052.60104^{\circ} 0158.30$ & 1.184 & 22 & Noria 2 Tuxcacuesco & $19^{\circ} 4046.80$ & $103^{\circ} 5834.10$ & 715 \\
\hline 3 & Puente La Callita-Arroyo & $20^{\circ} 0931.70104^{\circ} 0251.10$ & 1.178 & 23 & Noria 3 Tuxcacuesco & $19^{\circ} 4045.00$ & $103^{\circ} 5836.70$ & 715 \\
\hline 4 & Presa San Agustín & $20^{\circ} 0528.90104^{\circ} 1213.00$ & 1.362 & 24 & Río Tuxcacuesco en Tux & $19^{\circ} 4142.40$ & $103^{\circ} 5835.20$ & 726 \\
\hline 5 & Puente El Bado-río Ayutla & $20^{\circ} 0605.60104^{\circ} 1840.40$ & 1.346 & 25 & Río Ayuquila-Paso real & $19^{\circ} 3602.00$ & $103^{\circ} 5747.30$ & 696 \\
\hline 6 & Agua de riego-La Piñuela & $20^{\circ} 0057.80104^{\circ} 1607.60$ & 1.326 & 26 & Río Tuxcacuesco en Paso real & $19^{\circ} 3613.50$ & $103^{\circ} 5752.30$ & 705 \\
\hline 7 & Agua de riego-Unión de Tula & $19^{\circ} 5639.50104^{\circ} 1652.70$ & 1.367 & 27 & Río Armería en Paso real & $19^{\circ} 3603.50$ & $103^{\circ} 5735.30$ & 694 \\
\hline 8 & Puente El Corcovado & $19^{\circ} 5107.70104^{\circ} 1704.00$ & 909 & 28 & Manantial La Taza-San Pedro & $19^{\circ} 3549.40$ & $103^{\circ} 5749.10$ & 698 \\
\hline 9 & Río Ayuquila-Palo Blanco & $19^{\circ} 4431.50104^{\circ} 1040.70$ & 873 & 29 & Agua de riego-San Gabriel & $19^{\circ} 4512.60$ & $103^{\circ} 4555.60$ & 1.283 \\
\hline 10 & Agua de riego-El Aguacate & $19^{\circ} 4441.60104^{\circ} 0957.80$ & 865 & 30 & Río Comala & $19^{\circ} 1911.80$ & $103^{\circ} 4534.80$ & 605 \\
\hline 11 & Pozo La Ramada-Las Paredes & $19^{\circ} 4552.57104^{\circ} 1511.29$ & 870 & 31 & Villa de Álvarez-canal de riego & $19^{\circ} 1409.60$ & 600.90 & 460 \\
\hline 12 & Pozo Auxiliar-Las Paredes & $19^{\circ} 4550.56104^{\circ} 1452.91$ & 868 & 32 & Canal de riego-Coquimatlán & $19^{\circ} 1128.70$ & $103^{\circ} 5016.10$ & 302 \\
\hline 13 & Pozo El Ranchito-Las Paredes & $19^{\circ} 4550.56104^{\circ} 1559.04$ & 870 & 33 & Río Armería-Coquimatlán & $19^{\circ} 1138.60$ & $103^{\circ} 5010.10$ & 305 \\
\hline 14 & Noria La Ciénega & $19^{\circ} 5026.66104^{\circ} 0822.11$ & 904 & 34 & Manantial Los Amiales & $19^{\circ} 1001.40$ & $103^{\circ} 5001.10$ & 280 \\
\hline 15 & Agua de riego de traspatio & $19^{\circ} 5026.30104^{\circ} 0822.30$ & 904 & 35 & Afluente Armería-Coquimatlán & $19^{\circ} 1024.30$ & $103^{\circ} 4944.60$ & 2.952 \\
\hline 16 & Canal de riego-La Ciénega & $19^{\circ} 4955.60104^{\circ} 0805.10$ & 867 & 36 & Río Armería & $18^{\circ} 5716.60$ & $103^{\circ} 5641.90$ & 46 \\
\hline 17 & Río San Buenaventura & $19^{\circ} 4729.20104^{\circ} 0314.40$ & 790 & 37 & Río Armería-Boca de Pascuales & $18^{\circ} 5137.70$ & $103^{\circ} 5803.60$ & 3 \\
\hline 18 & Agua de riego-Tonaya & $19^{\circ} 4643.50104^{\circ} 5855.40$ & 837 & 38 & Agua de mar-Boca de Pascuales & $18^{\circ} 5138.70$ & $103^{\circ} 5802.50$ & 0 \\
\hline 19 & Río Tonaya & $19^{\circ} 4652.70103^{\circ} 5839.20$ & 817 & 39 & Río Tonaya-El Paso & $19^{\circ} 4928.19$ & $103^{\circ} 5639.92$ & 867 \\
\hline 20 & $\begin{array}{l}\text { Agua de riego-crucero } \\
\text { Tuxcacuesco }\end{array}$ & $19^{\circ} 4257.50103^{\circ} 5307.30$ & 1.000 & 40 & Noria Las Higueras-Tonaya & $19^{\circ} 4942.20$ & $103^{\circ} 5536.79$ & 926 \\
\hline
\end{tabular}




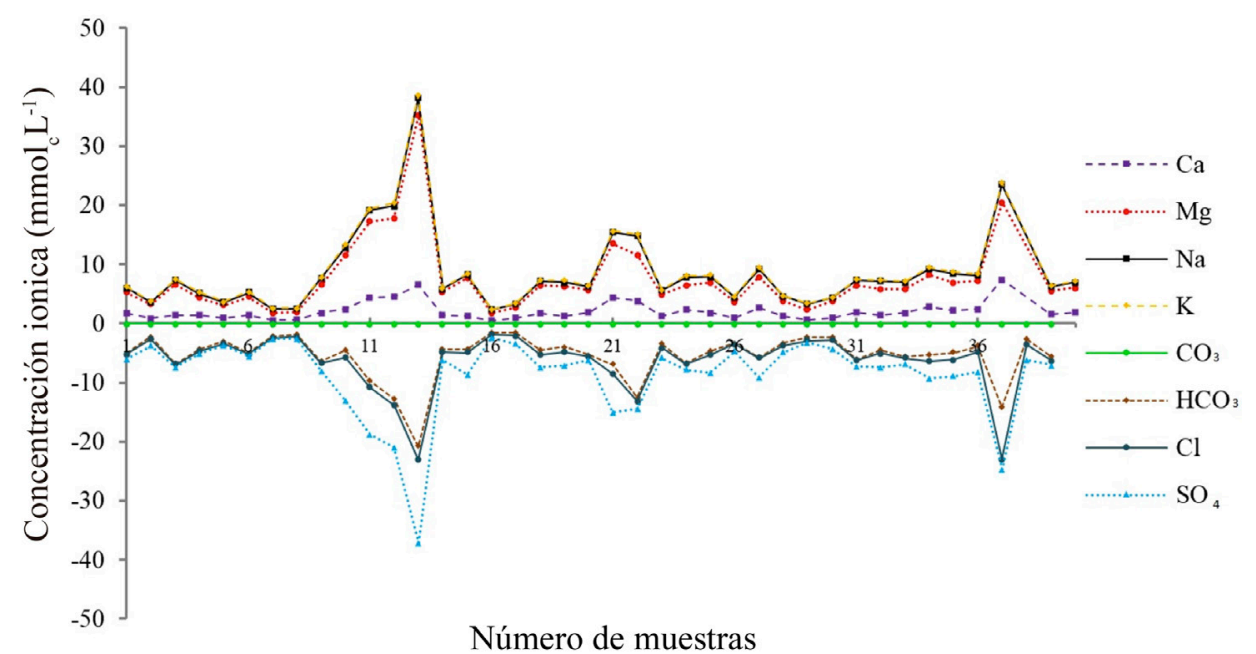

Figura 2. Distribución catiónica y aniónica de las aguas de riego del río Ayuquila-Tuxcacuesco-Armería.

del río Ayuquila-Tuxcacuesco-Armería, que nos permite observar con mayor claridad el predominio del anión bicarbonato y del catión magnesio. La presencia de magnesio y bicarbonatos en las aguas analizadas se debe a las reacciones de dilución, hidrólisis y óxido reducción que se llevan a cabo en el contacto de las rocas con el agua (Can-Chulim et al., 2011).

Las concentraciones más elevadas de $\mathrm{Mg}$ pertenecen a pozos, ríos y norias, el valor mayor de $35.229 \mathrm{mmol}_{\mathrm{c}} \mathrm{L}^{-1}$ para este elemento y para $\mathrm{HCO}_{3}$ de $20.810 \mathrm{mmol}_{\mathrm{c}} \mathrm{L}^{-1}$ fueron para el pozo El RanchitoLas Paredes; Las mínimas concentraciones de los mismos elementos fueron de $1.701 \mathrm{mmol}_{\mathrm{c}} \mathrm{L}^{-1}$ para el río en Unión de Tvla y $1.608 \mathrm{mmol}_{\mathrm{c}} \mathrm{L}^{-1}$ en el Canal de riego-La Ciénega. Las concentraciones elevadas de bicarbonato provocan el aumento del pH de la rizosfera y la disrupción del proceso de absorción de Fe causando clorosis férrica a una concentración de 41 meq L $\mathrm{L}^{-1}$ y disminuyendo el rendimiento en $7 \%$ en el caso del maíz (Shahabi et al., 2005).

Las concentraciones altas se encontraron en la parte media de la cuenca. La mayoría de las muestras que presentaron concentración iónica alta corresponden a pozos y norias.

Las descargas de aguas residuales agrícolas, el uso de fertilizantes y el contacto de las rocas con el agua, las que de acuerdo con su composición aportan diferentes iones, son algunas causas por las que se presenta la predominancia de bicarbonato y magnesio. En climas áridos, la existencia aun en pequeñas cantidades de bicarbonato sobre $\mathrm{Ca}$ + Mg en aguas de riego, llevarían a la formación de suelos sódicos (Sánchez et al., 2015).

La geología presente en el área de estudio, indica que las aguas están en contacto con materiales de origen volcánico los que son de baja concentración iónica, debido a que presentan baja solubilidad. Estas características influyen para que se presenten bajas concentraciones iónicas en algunos sitios de muestreo.

En la Figura 3, se presenta el pH y la conductividad eléctrica medidos en el agua de riego de la cuenca del río Ayuquila-Tuxcacuesco-Armería.

El pH de la solución tiene un efecto sobre la biodisponibilidad de la mayoría de los metales pesados al afectar el equilibrio entre la especiación metálica, solubilidad, adsorción e intercambio de iones en el suelo. En soluciones ácidas se produce una competencia de los iones $\mathrm{H}^{+}$con los cationes metálicos por los sitios de intercambio. Al aumentar el $\mathrm{pH}$, los metales pesados son removidos de la solución del suelo y adsorbidos por los coloides disminuyendo su biodisponibilidad. Por lo tanto, en suelos agrícolas con cargas variables, la retención de los cationes metálicos aumenta en la medida que el pH sea más alto y se reduce al acidificarse el suelo o el agua (Lamb et al., 2009).

En el agua analizada se encontraron valores de $\mathrm{pH}$ ligeramente alcalinos. La alcalinidad en el agua tanto natural como tratada, usualmente es causada por la presencia de iones carbonatos $\left(\mathrm{CO}_{3}^{2-}\right)$ y bicarbonatos $\left(\mathrm{HCO}^{3-}\right)$, asociados con 


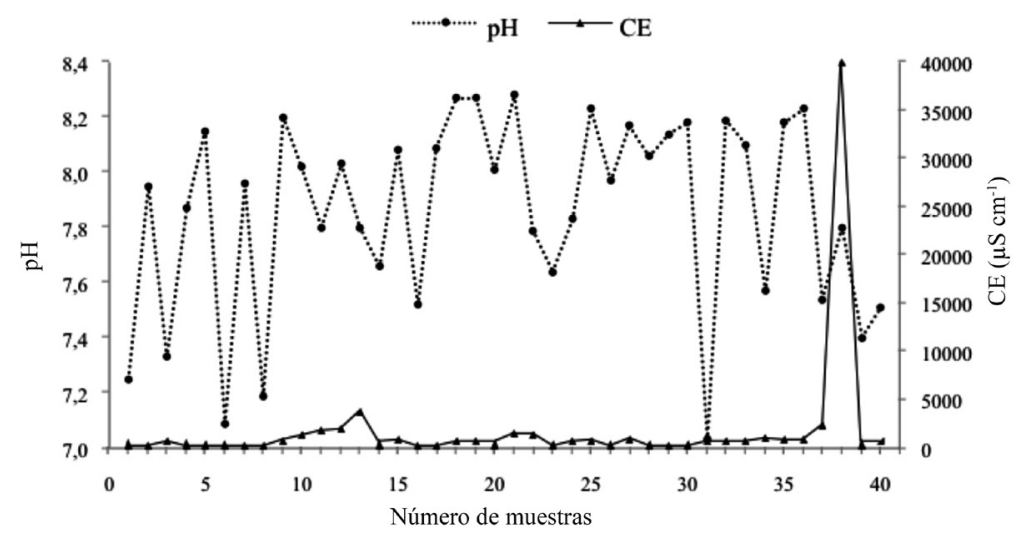

Figura 3. Conductividad eléctrica y pH medidos en las aguas de riego del río Ayuquila-Tuxcacuesco-Armería.

los cationes $\mathrm{Na}^{1+}, \mathrm{K}^{1+}, \mathrm{Ca}^{2+} \mathrm{y} \mathrm{Mg}^{2+}(\mathrm{Can}-\mathrm{Chulim}$ et al., 2008).

Las muestras presentaron un rango de $\mathrm{pH}$ entre 7 y 8,2 (promedio de 7,8), lo que indica que son ligeramente alcalinas. Los valores de $\mathrm{pH}$ son heterogéneos respecto al origen de las aguas (norias, pozos, ríos), no se presentaron valores similares en cada origen de las muestras.

Mientras que la conductividad eléctrica presentó un valor promedio de $1.882,94\left(\mu \mathrm{S} \mathrm{cm}^{-1}\right)$, lo que indica que el agua analizada, bajo el criterio de estos dos parámetros y desde el punto de vista agrícola, es recomendable de acuerdo con Ayers y
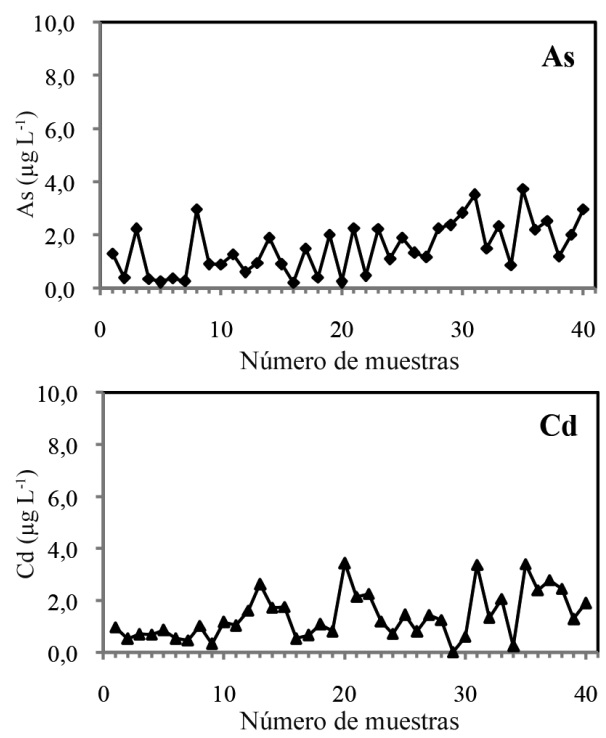

Westcot (1987), con restricción para una muestra que presentó elevado valor de $\mathrm{CE}$, debido a que procede directamente de agua marina.

Los parámetros pH y CE, están relacionados con el vigor de semillas y los porcentajes de germinación de semillas, esto según un estudio sobre germinación y crecimiento de plantas de Zea mays L. (Viloria et al., 2011), a mayor CE disminuye el porcentaje de germinación y el pH tuvo una fuerte asociación con el peso seco del vástago.

En la Figura 4, se presentan los resultados de la concentración de metales pesados en el agua muestreada.
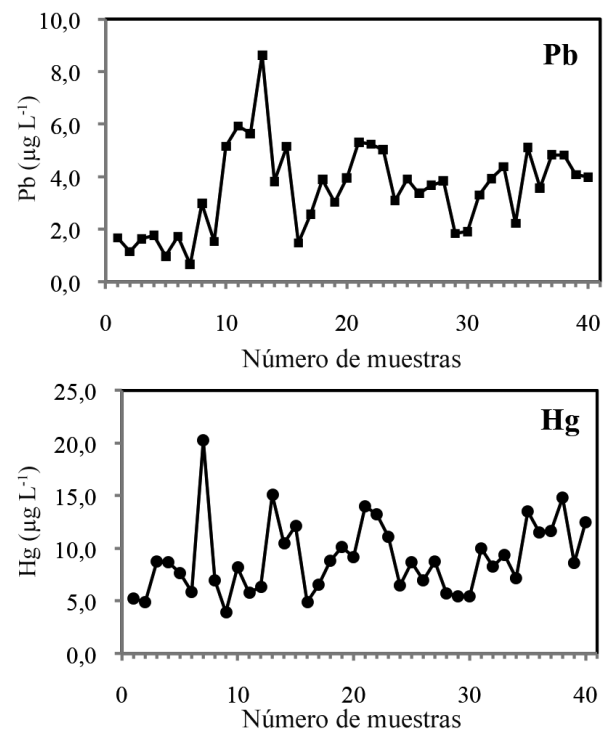

Figura 4. Concentración de metales pesados en las aguas de riego del río Ayuquila-Tuxcacuesco-Armería. 
Al comparar los valores obtenidos con los límites máximos permisibles establecidos por la SEMARNAT-NOM-001-ECOL-1996, 14 muestras superaron el límite máximo permisible de mercurio $\mathrm{Hg}\left(0,01 \mathrm{mg} \mathrm{L}^{-1}\right)$, para uso agrícola y urbano. Con respecto al criterio de EPA (1986), para aguas naturales, las 40 muestras superan el límite máximo permisible $\left(0,002 \mathrm{mg} \mathrm{L}^{-1}\right)$. En cuanto al contenido de plomo, 37 muestras superaron el límite máximo permisible establecido por la EPA (1986) $\left(0,0015 \mathrm{mg} \mathrm{L}^{-1}\right)$, para aguas naturales.

Las concentraciones de As y Cd están por debajo de los límites permisibles, por lo que el agua puede ser utilizada en el riego agrícola. Sin embargo, se debe tener restricción de uso donde superó los límites de concentración de $\mathrm{Pb}$ y $\mathrm{Hg}$.

Mancilla-Villa et al. (2012) realizaron un estudio en los ríos, embalses y manantiales de Puebla y Veracruz sobre el contenido de metales pesados donde el mercurio superó el límite máximo permisible señalado por la SEMARNATNOM-001-ECOL-1996 para uso urbano. Para el criterio de EPA (1986), se encontró que 1, 30, 15 y $20 \%$ de las muestras analizadas supera el límite permisible para $\mathrm{As}, \mathrm{Pb}, \mathrm{Cd}$ y $\mathrm{Hg}$, respectivamente. La zona objeto de estudio presenta características

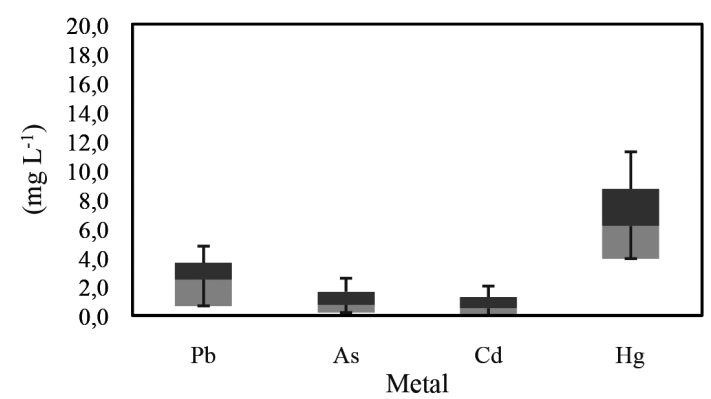

Figura 5. Diagrama de cajas que muestra la distribución cuartil del As y los metales pesados en el agua analizada.

similares, esta diferencia de concentraciones se debe a la intensa actividad agrícola, industrial y urbanización, en los diferentes sitios de muestreo.

La distribución del contenido de As y metales pesados en el agua, se presenta en la Figura 5. El Cd presentó los valores más bajos, el límite superior fue de $0,0034 \mathrm{mg} \mathrm{L}^{-1}$ Por su parte el $\mathrm{Hg}$ mostró los más altos contenidos alcanzando valores de hasta $0,02 \mathrm{mg} \mathrm{L}^{-1}$

Respecto a salinidad y sodicidad en la Figura 6, se encuentran representados los valores para la clasificación de agua en cuanto a dichos índices.

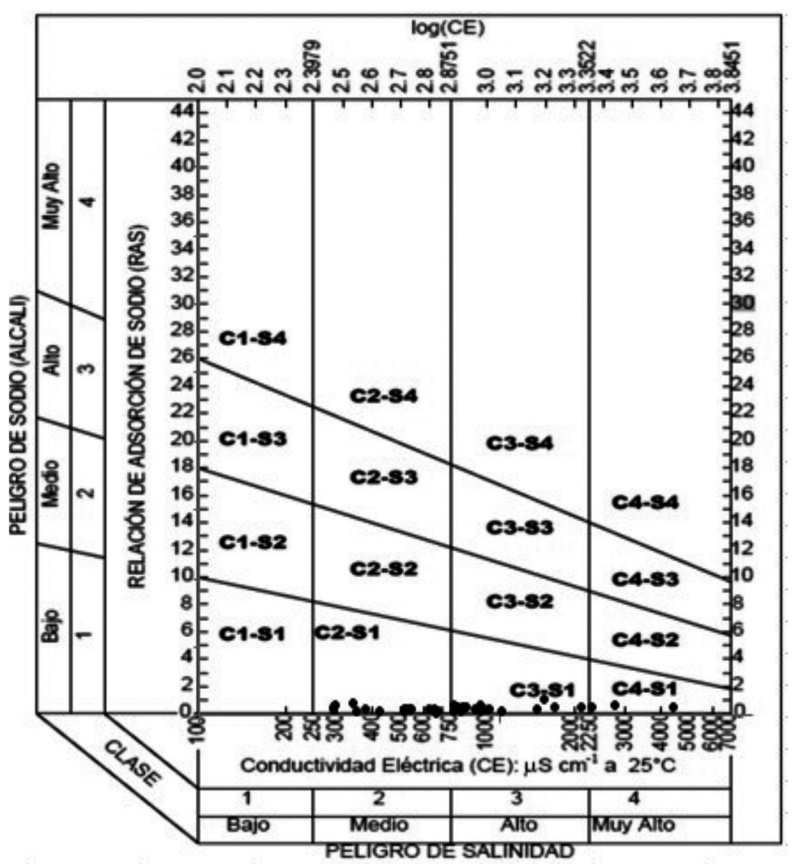

Figura 6. Diagrama de Richards para clasificación en salinidad y sodicidad, de muestras de agua. 


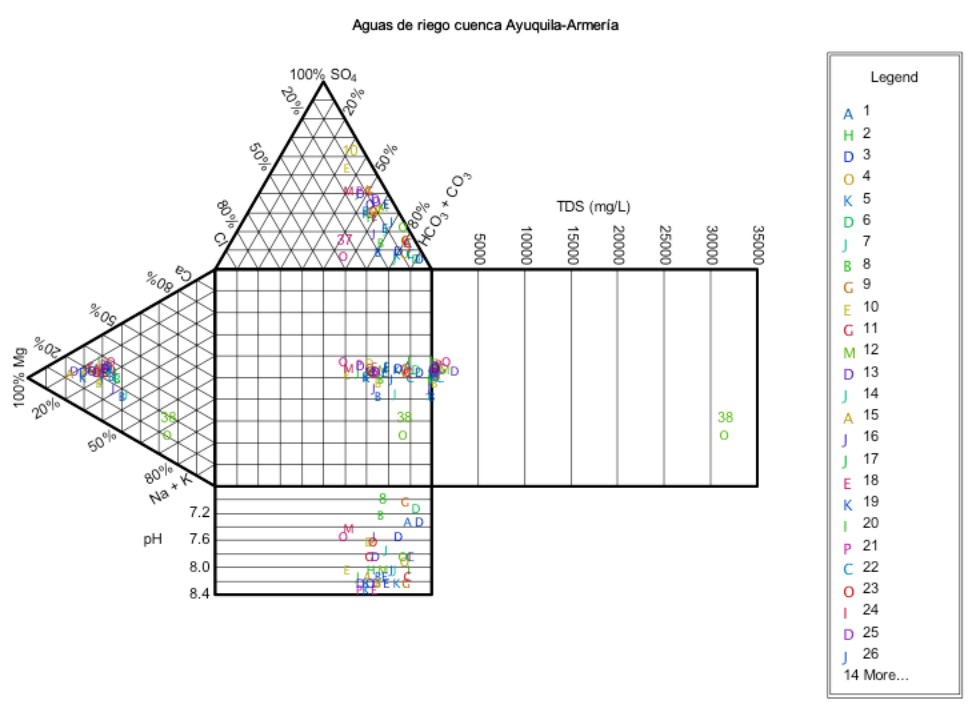

Figura 7. Diagrama de Durov para las aguas de riego del río Ayuquila-Tuxcacuesco-Armería

Todas las muestras se encuentran dentro de la clasificación S1, que se refiere en aguas con bajo peligro de sodicidad, esto se explica en relación a las composiciones iónicas, las que presentan concentraciones importantes de $\mathrm{Ca}$ y $\mathrm{Mg}$ en comparación a las de sodio. En cuanto a la salinidad las muestras se ubican dentro de $\mathrm{C} 2, \mathrm{C} 3$ y dos en $\mathrm{C} 4$, que corresponden a aguas con medio, alto $\mathrm{y}$ muy alto peligro de salinidad, respectivamente (Richards, 1973).

A continuación se presenta el diagrama de Durov (Figura 7) en donde se muestra la composición iónica de las aguas del riego del río Ayuquila-Tuxcacuesco-Armería, la cantidad de sólidos totales disueltos y el $\mathrm{pH}$.

Los sólidos totales disueltos en su mayoría se encuentran en una concentración alrededor de $500 \mathrm{mg} \mathrm{L}^{-1}$ y 2.500 excepto por la muestra de agua de mar Boca de Pascuales que presentó una elevada concentración. De acuerdo con Ayers y Wescot (1987) las aguas que presentan valores entre 450-2000 mg L-1, para sólidos totales disueltos, tienen un grado de restricción ligera a moderada en cuanto a su uso para riego. El pH resultó alcalino debido a la presencia de bicarbonatos (Can-Chulim et al., 2011).

Existe una correlación directa entre la conductividad eléctrica y la concentración de sólidos disueltos totales para cuerpos de agua dulce y salobre (Can-Chulim et al., 2014). Condición que puede observarse en los resultados del presente estudio, las muestras con los mayores valores de CE, coinciden con las que presentan valores elevados de $\mathrm{Mg}$ y $\mathrm{HCO}_{3}$. Esta correlación no aplica para ambientes hipersalinos, donde la conductividad es afectada por la composición específica de iones presentes en el agua (Mancilla-Villa et al., 2012).

\section{Conclusiones}

El pH y la conductividad eléctrica de la mayoría del agua analizada, no limitan el uso urbano o para riego agrícola especialmente para cultivos tolerantes y semitolerantes, no así para los sensibles, por lo que es recomendable para las actividades mencionadas. De acuerdo con la concentración iónica, las aguas se clasifican como bicarbonatadas-magnésicas.

El Hg superó los límites máximos permisibles establecidos por la SEMARNAT-NOM-001ECOL-1996 para 35\% de las muestras. En cuanto al criterio de SEDUE (1989), no existe problema en el uso del agua muestreada para recreación, como fuente de agua potable y en riego.

De acuerdo con los límites permisibles de la EPA (1986), 100\% de los cuerpos de agua analizados exceden las concentraciones de $\mathrm{Hg}$ y $92,5 \%$ de $\mathrm{Pb}$.

Con respecto a salinidad cerca de $80 \%$ de las aguas se clasificaron como buenas, $18 \%$ como condicionadas y $2 \%$ restante como no recomendables, en cuanto a sodicidad, las aguas analizadas son aptas para uso agrícola. 


\section{Literatura Citada}

APHA

1995. Standard methods for examination of water and wastewater. APHA (American Public Health Association), WWA (American Water Works Association), WPCF (Water Pollution Control Federation), Washington D.C., USA. $1035 \mathrm{pp}$.

Can-Chulim, Á.; Ramírez-Ayala, C.; Ortega-Escobar, H.; Trejo López C. y Cruz-Díaz. J.

2008. Evaluación de la relación de adsorción de sodio en las aguas del río Tulancingo, estado de Hidalgo, México. Terra Latinoamericana, 26: 243-252.

Can-Chulim, Á.; Ortega-Escobar, H. M.; García-Calderón, N. E.; Reyes-Ortigoza, A. L.; González-Hernández, V. A.; Flores-Román, D.

2011. Origen y calidad del agua subterránea en la cuenca Oriental de México. Terra Latinoamericana, 29 (2): 189-200.

Can-Chulim, Á.; Ortega-Escobar, H.M.; Sánchez-Bernal, E.

I.; Cruz-Crespo, E.

2014. Calidad del agua para riego en la Sierra Norte de Puebla, México. Tecnología y Ciencias del Agua, 5 (5): 77-96.

\section{CONAGUA}

2007. Estadísticas del agua en México, edición 2007. Comisión Nacional del Agua, Secretaría del Medio Ambiente y Recursos Naturales. México.

Duffus, J.H.

2002.'Heavy metals" a meaningless term? (IUPAC Technical Report). Pure and Applied Chemistry, 74: 793-807. EPA

1986. Gold Book of Quality Criteria for Water. (Environmental Protection Agency) EPA 440/5-86-001. EUA. 477 p.

Lamb, D.T.; Ming, H.; Megharaj, M.; Naidu, R.

2009. Heavy metal $(\mathrm{Cu}, \mathrm{Zn}, \mathrm{Cd}$ and $\mathrm{Pb})$ partitioning and bioaccessibility in uncontaminated and long-term contaminated soils. Journal of Hazardous Materials, 171: 1150-1158.

Llugany, M.; Tolrá, R.; Poschnrieder, C.; Barceló, J.

2007. Hiperacumulación de metales: ¿Una ventaja para la planta y para el hombre? Rev. Ecosistemas., 16: 4-9.

Mancera-Rodríguez, N.J.; Alvárez-León, R.

2006. Estado del conocimiento de las concentraciones de mercurio y otros metales pesados en peces dulceacuícolas de Colombia. Acta Biológica Colombiana, 11: 3-23.

Mancilla-Villa O.R.; Ortega E., H.M.; Ramírez A., C.; Uscanga

M., E.; Ramos B., R.; Reyes O., A.L.

2012. Metales pesados totales y arsénico en el agua para riego de Puebla y Veracruz, México. Rev. Int. Contam. Ambie. 28 (1): 39-48.

Martí, L.; Filippini, M.F.; Salcedo, C.; Drovandi, A.; Troilo, S.; Valdés, A.

2011. Evaluación de metales pesados en suelos de los oasis irrigados de la Provincia de Mendoza: I. Concentraciones totales de $\mathrm{Zn}, \mathrm{Pb}, \mathrm{Cd}$ y Cu. Rev. FCA UNCUYO, 43: 203-221.

Méndez R., M.; Armienta H., M.A.

2012. Distribución de $\mathrm{Fe}, \mathrm{Zn}, \mathrm{Pb}, \mathrm{Cu}, \mathrm{Cd}$ y As originada por residuos mineros y aguas residuales en un transecto del río Taxco en Guerrero, México. Rev. Mex. Cienc. Geol., 29 (2): 450-462.
Méndez, J.P.; Ramírez, C.A.G.; Gutiérrez, A.D.R.; García, F.P. 2009. Contaminación y fitotoxicidad en plantas por metales pesados provenientes de suelos y agua [plant contamination and phytotoxicity due to heavy metals from soil and water]. Tropical and Subtropical Agroecosystems, 10 (1): 29-44.

Mor, F.; Ceylan, S.

2008. Cadmium and lead contamination in vegetables collected from industrial, traffic and rural areas in Bursa Province, Turkey. Food Additives \& Contaminants: Part A, 25 (5): 611-615.

Secretaría de Medio Ambiente, Recursos Naturales y Pesca. NOM-001-ECOL

1996. Norma oficial mexicana que establece los límites máximos permisibles de contaminantes en las descargas de aguas residuales a los sistemas de alcantarillado urbano o municipal. Publicada en el diario oficial de la federación el 03 de junio de 1998, 20 p.

Prieto, M.J.; González R., C.A.; Román G., A.D.; Prieto, G.F. 2009. Contaminación y fitotoxicidad en plantas por metales pesados provenientes de suelos y agua. Tropic. Subtropic. Agroecosyst, 10: 29-44.

Richards, L.A. (Editor).

1973. Suelos Salinos y Sódicos. Personal del Laboratorio de Salinidad de los Estados Unidos de América. Manual de Agricultura $\mathrm{N}^{\mathrm{o}} 60$, p. 172.

Sánchez, D.; Barberá, J.A.; Mudarra, M.; Andreo, B.

2015. Hydrogeochemical tools applied to the study of carbonate aquifers: examples from some karst systems of Southern Spain. Environmental Earth Sciences, 74 (1), 199-215.

Sarabia M., I.F., Cisneros A., R.; Aceves de A., J.; Durán G.,

H.M.; Castro L., J.

2011. Calidad del agua de riego en suelos agrícolas y cultivos del Valle de San Luis Potosí, México. Rev. Int. Contam. Ambie. 27 (2): 103-113.

SEDUE

1989. Acuerdo por el que se establecen los Criterios Ecológicos de Calidad de Agua CE-CCA-001/89. Secretaría de Desarrollo Urbano y Ecología. Publicado en el Diario Oficial de la Federación del 2 de diciembre de 1989. Tomo CDXXX, No 9. México, D.F.

Shahabi, A.; Malakouti, M. J.; Fallahi, E.

2005. Effects of Bicarbonate Content of Irrigation Water on Nutritional Disorders of Some Apple Varieties. Journal of plant nutrition, 28 (9): 1663-1678.

Velázquez, M.M.A.; Ortega E.; A. Martínez G.; Kohashi S.; García C.N.

2002. Relación funcional PSI-RAS en las aguas residuales y suelos del Valle del Mezquital, Hidalgo, México. Terra. 20 (4): 459-464.

Viloria, H.; Méndez-Natera, J.R.

2011. Relación entre la conductividad eléctrica, pH del agua de remojo, germinación de semillas y crecimiento de plántulas de maíz (Zea mayz L.) bajo dos condiciones experimentales. Scientia Agropecuaria, 2 (4): 213-228. 
